\title{
RDGN-based predictive model for the prognosis of breast cancer
}

\author{
Bing Dong ${ }^{1 \dagger}$, Ming $\mathrm{Yi}^{2 \dagger}$, Suxia LuO ${ }^{3}$, Anping $\mathrm{Li}^{3^{*}}$ and Kongming $\mathrm{Wu}^{2,3^{*}}$ (])
}

\begin{abstract}
Background: Breast cancer is the most diagnosed malignancy in females in the United States. The members of retinal determination gene network (RDGN) including DACH, EYA, as well as SIX families participate in the proliferation, apoptosis, and metastasis of multiple tumors including breast cancer. A comprehensive predictive model of RDGN might be helpful to herald the prognosis of breast cancer patients.

Methods: In this study, the Gene Expression Ominibus (GEO) and Gene Set Expression Analysis (GSEA) algorithm were used to investigate the effect of RDGN members on downstream signaling pathways. Besides, based on The Cancer Genome Atlas (TCGA) database, we explored the expression patterns of RDGN members in tumors, normal tissues, and different breast cancer subtypes. Moreover, we estimated the relationship between RDGN members and the outcomes of breast cancer patients. Lastly, we constructed a RDGN-based predictive model by Cox proportional hazard regression and verified the model in two separate GEO datasets.

Results: The results of GSEA showed that the expression of DACH1 was negatively correlated with cell cycle and DNA replication pathways. On the contrary, the levels of EYA2 and SIX1 were significantly positively correlated with DNA replication, mTOR, and Wnt pathways. Further investigation in TCGA database indicated that DACH1 expression was lower in breast cancers especially basal-like subtype. In the meanwhile, SIX1 was remarkably upregulated in breast cancers while EYA2 level was increased in Basal-like and Her-2 enriched subtypes. Survival analyses demonstrated that DACH1 was a favorable factor while EYA2 and SIX1 were risk factors for breast cancer patients. Given the results of Cox proportional hazard regression analysis, two members of RDGN were involved in the present predictive model and patients with high model index had poorer outcomes.
\end{abstract}

Conclusion: This study showed that aberrant RDGN expression was an unfavorable factor for breast cancer. This RDGN-based comprehensively framework was meaningful for predicting the prognosis of breast cancer patients.

Keywords: Breast cancer, RDGN, DACH, EYA, SIX, Predictive model

*Correspondence: li_anping@yahoo.com; kmwu2005@yahoo.com ${ }^{+}$Bing Dong and Ming Yi are contributed equally to this work

2 Department of Oncology, Tongji Hospital of Tongji Medical College,

Huazhong University of Science and Technology, Wuhan 430030, China

${ }^{3}$ Department of Medical Oncology, The Affiliated Cancer Hospital

of Zhengzhou University \& Henan Cancer Hospital, Zhengzhou 450008, China

Full list of author information is available at the end of the article

\section{Introduction}

In the United States, breast cancer is the most commonly diagnosed cancer and second leading cause of cancerrelated death in women $[1,2]$. According to the presence or absence of molecular biomarkers, breast cancer could be categorized into three major subtypes: Luminal (hormone receptor positive and Her-2 negative), Her-2-enriched (Her-2 amplified), Basal-like (hormone receptor negative and Her-2 negative) [3]. The molecular typing of breast cancer is a huge breakthrough in cancer theranostics which not only helps to predict the prognosis of

(c) The Author(s) 2020. This article is licensed under a Creative Commons Attribution 4.0 International License, which permits use, sharing, adaptation, distribution and reproduction in any medium or format, as long as you give appropriate credit to the original author(s) and the source, provide a link to the Creative Commons licence, and indicate if changes were made. The images or other third party material in this article are included in the article's Creative Commons licence, unless indicated otherwise in a credit line to the material. If material is not included in the article's Creative Commons licence and your intended use is not permitted by statutory regulation or exceeds the permitted use, you will need to obtain permission directly from the copyright holder. To view a copy of this licence, visit http://creativeco mmons.org/licenses/by/4.0/. The Creative Commons Public Domain Dedication waiver (http://creativecommons.org/publicdomain/ zero/1.0/) applies to the data made available in this article, unless otherwise stated in a credit line to the data. 
patients, but also effectively guides the following treatment schedule [4-6]. Among the three breast cancer subtypes, Basal-like breast cancer has the poorest clinical outcome and the highest probability to reoccur than other two subtypes of cancers [7]. Apart from molecular typing, tumor DNA sequencing is also an important reference for treatment decision. Patients with germline mutations in $B R C A$ might benefit from PARP inhibitor treatment while patients harboring alterations in $E R B B-2$ or ESR 1 are more likely to develop resistance to standard therapies [8-13].

As a highly conservative signaling pathway, the retinal determination gene network (RDGN) was originally found to regulate Drosophila eye specification. Then, RDGN was reported to participate in the organ development in mammals [14]. At present, it has been well established that aberrantly expressed RDGN signals involve in the proliferation, apoptosis, stemness, and metastasis of cancer cells [15]. It has been known that RDGN comprises multiple members: dac/Dach (dominant suppressor of ellipse), eya/Eya (tyrosine phosphatase eyes absent), so/Six (Six family transcription factor sine oculis), as well as ey/toy (Pax6-like homeodomain proteins) [16]. In the main components of RGDN, DACH family generally plays a role as tumor suppressor while EYA and SIX families most likely act as oncogenes [17-21]. However, there are contrary reports on RDGN's function in different cancers. For example, DACH1 protein levels were increased with the invasiveness of the ovarian cancer and subcellular distribution of DACH1 changed from nucleus in normal tissue to cytoplasm in cancer [22]. As a negative regulator of Wnt pathway, SIX3 inhibited breast cancer carcinogenesis and metastasis through recruiting the LSD1/NuRD complex [23]. In consistent with this experimental study, expression profile analysis indicated that high SIX3 mRNA level was a protective factor for OS and RFS of basal-like breast cancer patients [24]. Several studies proved that EYA4 behaved as a tumor suppressor and associated with favorite prognosis in hepatocellular carcinoma and pancreatic ductal adenocarcinoma [25, 26]. In summary, multiple studies suggested that members of RDGN family played distinct roles depending on the cancer type. Our previous studies showed that RDGN was dysregulated in tumors with a coordinated fashion: downregulated DACH1 in accompany with upregulated EYA1 and SIX1 in tumors [24, 27, 28].

Several groups attempted to address the prognostic and therapeutic response value of $\mathrm{DACH} 1$ in breast cancer. Machine learning methods such as Artificial Neural Networks have been utilized to identify biomarkers of breast cancer. Using Artificial Neural Networks approach, Powe et al. [29] found that DACH1 had a positive association with ER and exerted a strong influence on ER associated markers. Consisting with our study, nuclear DACH1 expression was observed in normal and Luminal breast cancer tissues. Patents with high expression of DACH1 demonstrated longer survival and disease-free interval as well as reduced metastasis risk [29]. However, prognostic value of DACH1 was not independent of clinical stage and Nottingham Prognostic Index [29]. Aromatase inhibitors (AI) are standard adjuvant treatment for postmenopausal luminal A subtype breast cancer [30]. However, resistance is still a major clinical problem for improving long term survival. Thomsen et al. [30] performed global gene expression analysis to measure gene expression profile of $23 \mathrm{ER}$ positive breast cancer patients treated with adjuvant AI and collected follow-up information for relapse. Twenty-six genes including DACH1 were shown to exhibit altered expression in tumors from patients with relapse versus non-relapse. Ingenuity pathway analysis indicated DACH1 was linked with cyclin D1, cyclin A1, NRG family, and PLC1 [30]. It is interesting to mention that four methylation markers (RASGRF1, CPXM1, HOXA10, and DACH1) in circulating cell-free DNA could discriminate cancer from normal with high sensitivity $(0.86)$ and specificity (0.83) in early breast cancer [31]. Among the 4 genes, RASGRF1 and CPXM1 represented common breast cancer marker, while HOXA10 and DACH1 represented luminal-dominant marker and triple negative dominant marker, respectively. CpG islands of those 4 genes were located in the promoter region and associated with $\mathrm{H} 3 \mathrm{~K} 4 \mathrm{Me} 3$ enrichment. Those studies clearly indicated that the unbalanced RDGN status might be a useful biomarker to predict the prognosis of breast cancer patients. In this study, based on the results of bioinformatics analyses, we combined several members of RDGN and some well-accepted prognostic indicators to construct a predictive model for breast cancer patients.

\section{Methods \\ Gene expression profiles}

Two gene expression profiles (GSE25066 and GSE1456) were downloaded from Gene Expression Ominibus (https://www.ncbi.nlm.nih.gov/geo/). GSE25066 dataset was uploaded by Hatzis et al. [32] consisting of 508 breast cancer samples. GSE1456 dataset with 159 breast cancer samples was uploaded by Pawitan et al. [33]. Both GSE25066 and GSE1456 datasets were based on Affymetrix Human Genome U133A Array Platform. The pre-processed gene expression data of The Cancer 
Genome Atlas (TCGA) were obtained from UCSC Xena (https://xena.ucsc.edu/).

\section{Gene set enrichment analysis (GSEA)}

GSEA software (version: 4.0.3) was utilized for enrichment analysis [34]. Gene set databases c2.cp.kegg. v7.0.symbols.gmt and c5.all.v7.0.symbols.gmt were used for KEGG pathways and GO-terms enrichment analyses. We set gene size $>15$, False discovery rate $<0.25, \mathrm{P}<0.05,|\mathrm{NES}|>1$ as positive criteria.

\section{Survival analysis}

The survival analysis was conducted by online analysis tool Kaplan-Meier-plotter (https://kmplot.com/analy sis/). Kaplan-Meier-plotter could assess the effect of over 54,000 genes on the prognosis of multiple cancers [35]. In this study, we used the overall survival (OS), relapse-free survival (RFS), distant metastasis-free survival (DMFS), and post-progression survival (PPS) as main parameters to evaluate to the influence of RDGN on the outcomes of breast cancer patients.

\section{Predictive model construction and verification}

The three members of RDGN (DACH1, EYA2, and SIX1) and several well-established molecular biomarkers were included in Cox univariate regression analysis. According to the results of Cox univariate regression analysis, we selected RDGN members with statistical significance $(\mathrm{P}<0.10)$ and other verified molecular biomarkers to construct Cox proportional hazards model. Then, we used time-dependent ROC curves to evaluate the accuracy and specificity of the risk model. Lastly, we used K-M plotter curves to assess the difference in prognosis between patients with high risk index (33.3 in the top percentile) and low risk index (33.3 in the last percentile). GSE1456 was the training set and GSE25066 was the validation set.

\section{Statistical analysis}

The comparisons between different groups were conducted by Students't test. All statistical results with a $P$ value $<0.05$ were considered significant. The survival curves were performed by Kaplan-Meier curves with log-rank test. Statistical analyses were conducted with Graphpad Prism 8.0 and R software (version 3.6.0 with package survminer and survivalROC).

\section{Results}

\section{KEGG pathways and GO-terms enrichments}

Based on dataset GSE25066, we separately analyzed the difference of gene profiles for high and low expression of DACH1, EYA2 and SIX1. The results of GSEA showed that DACH1 level was negatively related with cell cycle, DNA replication, mismatch repair, and homologous recombination pathways (Fig. 1a-d). The GO-terms enrichment demonstrated that DACH1 level was negatively correlated with cell cycle G1/S phase translation, meiotic chromosome segregation but positively related to mammary gland morphogenesis processes (Fig. 2a-d). On the contrary, EYA2 level was positively related with cell cycle, DNA replication, Wnt signaling pathway, regulation of action cytoskeleton pathways (Fig. 1e-h). Correspondingly, the GO-terms enrichment indicated that EYA2 was positively correlated to cell cycle G1/S phase translation, NF- $\mathrm{KB}$ pathway, Wnt pathway, DNA repair processes (Fig. 2e-j). Similar to EYA2, SIX1 level was positively correlated to DNA replication, mTOR pathway, cell adhesion molecules, and antigen presentation pathways (Fig. 1i-l). The results of SIX1 GO-terms enrichment analysis revealed that SIX1 was negatively related to anti-tumor immune response and $\mathrm{T}$ cell activity but positively correlated to embryonic development (Fig. 2k-p).

\section{Decreased DACH1 predicting poor prognosis of breast cancer}

Based on the expression profiles from TCGA database, we compared the DACH1 level between normal breast tissues and breast cancer samples. The results showed that DACH1 level in tumor tissues was significantly lower than normal tissues $(\mathrm{P}<0.05)$ (Fig. 3a). Further analysis showed that $\mathrm{DACH} 1$ was remarkably downregulated in Basal-like subtype compared with Luminal A or $\mathrm{B}$ or Her-2 subtypes $(\mathrm{P}<0.0001)$ (Fig. $3 \mathrm{~b})$. The following survival analyses confirmed that high DACH1 level was a favorable factor for patients' prognosis. Patients with higher DACH1 (above median level) had longer OS $(\mathrm{HR}=0.66, \mathrm{P}=0.01)$ (Fig. 3c), RFS $(\mathrm{HR}=0.71, \mathrm{P}<0.0001)$ (Fig. 3d), DMFS (HR $=0.64, P=0.0062$ ) (Fig. 3e), and PPS $(\mathrm{HR}=0.59, \mathrm{P}=0.0039)$ (Fig. 3f).

\section{Increased EYA2 and SIX1 predicting poor prognosis of breast cancer}

The expression data of EYA2 and SIX1 were extracted from TCGA database. EYA2 level was lower in cancer samples than normal breast samples while SIX1 level was higher in cancer samples than normal breast samples (Figs. 4a and 5a). Moreover, among all breast cancer subtypes, Basal-like subtype possessed the highest EYA2 level (Fig. 4b). Contrarily, the SIX1 level in Basal-like subtype was markedly lower than Luminal and Her2-enriched subtypes (Fig. 5b). Generally, both EYA2 and SIX1 were risk factors for prognosis of breast cancer patients. Patients with high EYA2 (above median value) had shorter $\mathrm{OS}(\mathrm{HR}=1.28, \mathrm{P}=0.024)$ 


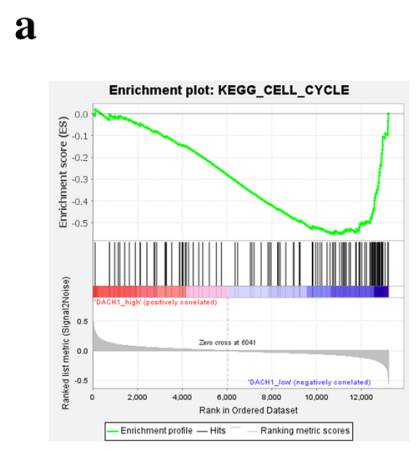

e

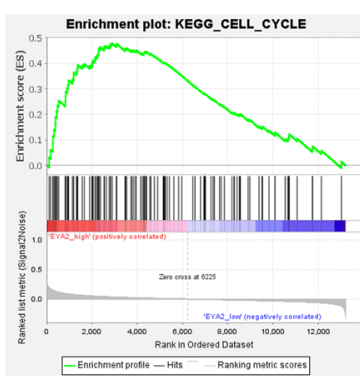

i

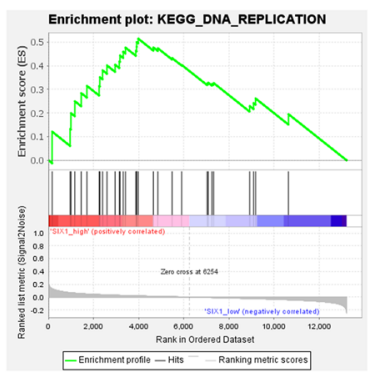

b

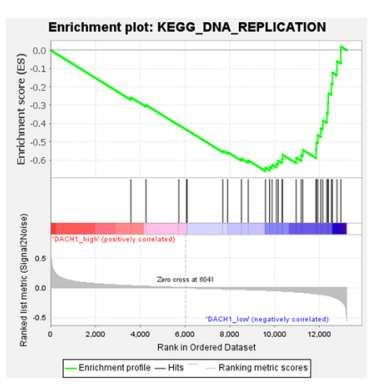

f

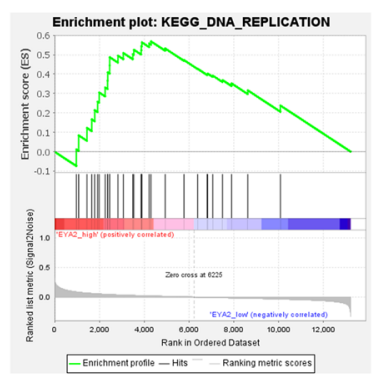

j

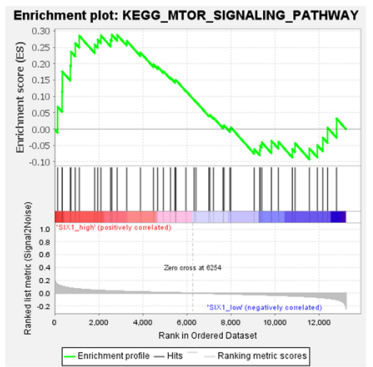

c

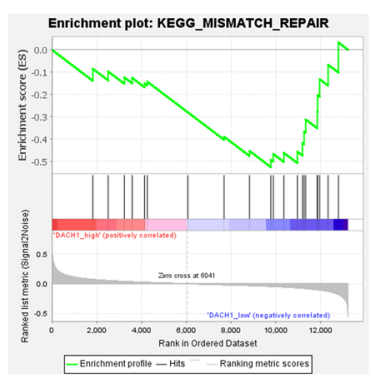

g

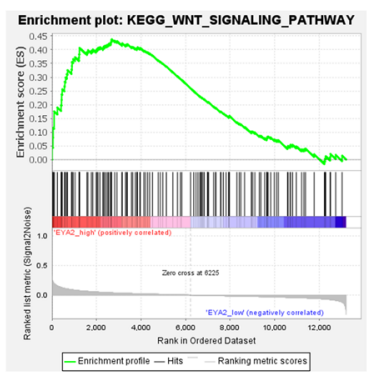

k

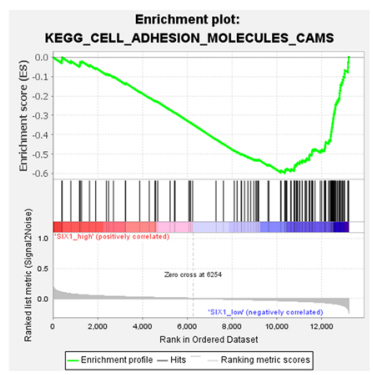

d

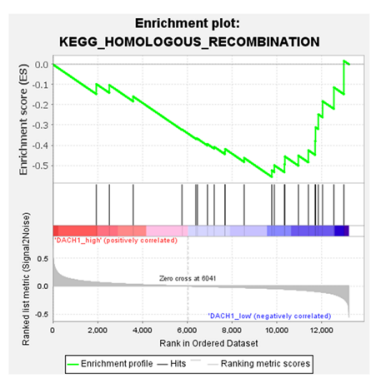

h

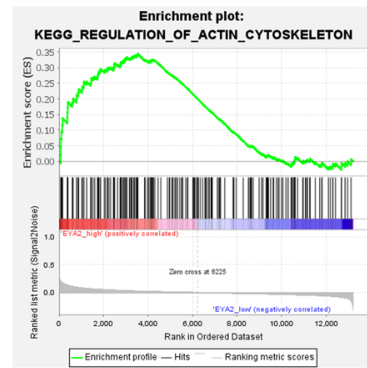

I

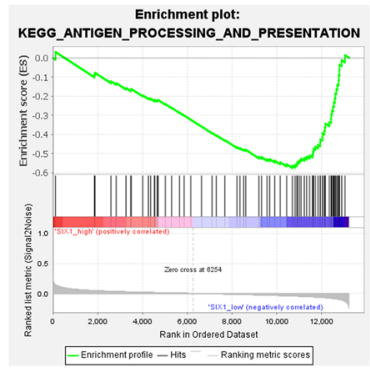

Fig. 1 The results of GSEA (KEGG pathways). The gene profiles of samples with DACH1 low expression were significantly enriched in cell cycle (a), DNA replication (b), mismatch repair (c), and homologous recombination (d); The gene profiles of samples with EYA2 high expression were significantly enriched in cell cycle (e), DNA replication (f), Wnt signaling pathway $(\mathbf{g})$, and regulation of actin cytoskeleton (h); The gene profiles of samples with SIX1 high expression were significantly enriched in DNA replication (i) and mTOR pathway (j); The gene profiles of samples with SIX1 low expression were significantly enriched in adhesion molecules (k) and antigen processing and presentation pathway (I)

(Fig. 4c), RFS (HR=1.2, $\mathrm{P}=0.00084$ ) (Fig. 4d), DMFS $(\mathrm{HR}=1.4, \mathrm{P}=0.00062)$ (Fig. 4e), but had no effect on PPS ( $\mathrm{HR}=1.07, \mathrm{P}=0.58$ ) (Fig. 4f). Similarly, patients with higher SIX1 (above median value) had poorer OS $(\mathrm{HR}=1.38, \mathrm{P}=0.0034)$ (Fig. 5c), RFS $(\mathrm{HR}=1.2$, $\mathrm{P}=0.0011) \quad$ (Fig. $\quad 5 \mathrm{~d}), \quad$ DMFS $\quad(\mathrm{HR}=1.21, \quad \mathrm{P}=0.054)$ (Fig. 5e), as well as PPS ( $\mathrm{HR}=1.33, \mathrm{P}=0.021)$ (Fig. 5f).

\section{Predictive model construction and verification}

According to the clinical information and gene matrix of GSE1456, we performed Cox univariate regression analysis to select prognosis indicators from RDGN. Eventually, we utilized DACH1 $(\mathrm{HR}=0.758, \mathrm{P}=0.083)$,
SIX1 $(\mathrm{HR}=1.365, \mathrm{P}=0.036)$, as well as other previously verified molecular factors to construct a predictive model for potential relapse of breast cancer patients (Fig. 6a). Besides, we referred to the gene set designed by Pawitan et al. [33] and involved ERBB2, ESR, Cyclin E2, as well as TOP2A in our model to increase predictive power. By Cox proportional hazard model, we constructed the risk index $=-0.186^{*} \mathrm{DACH} 1+0.287^{*}$ SIX1 $+0.041^{*}$ ERBB2 $-0.044^{*}$ ESR1 (ESR) + 0.108*Cyclin E2 $+0.505 *$ TOP2A. Then, the time-dependent ROC curves showed that this index could effectively distinct patients with high relapse risk within 3, 5, and 8 years (AUC $>0.7$ in all conditions) (Fig. 6b-d). By K-M 


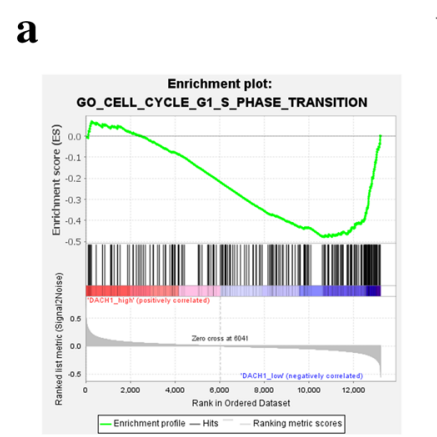

b

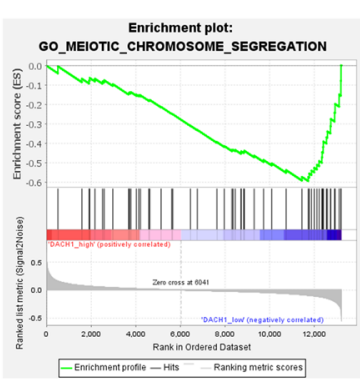

e

Enrichment plot:
GO_POSITIVE_REGULATION_OF_G1_S_TRANSITION_OF

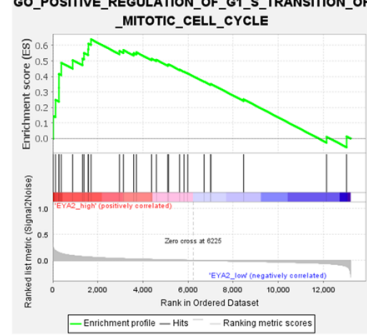

i

Enrichment plot:
GO_POSITIVE_REGULATION_OF_WNT_SIGNALING_PAT

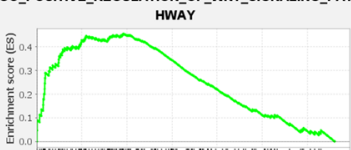

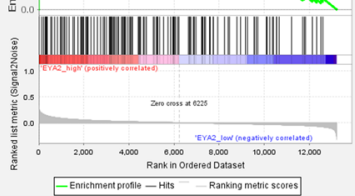

m

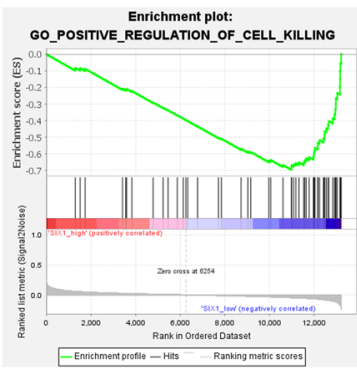

f

Enrichment plot:
GO_CELL_CYCLE_G1_S_PHASE_TRANSITION
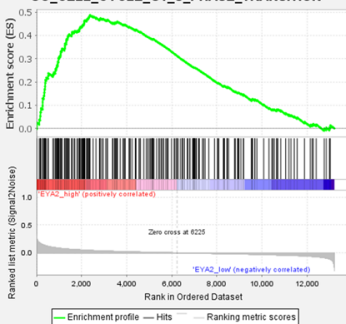

j

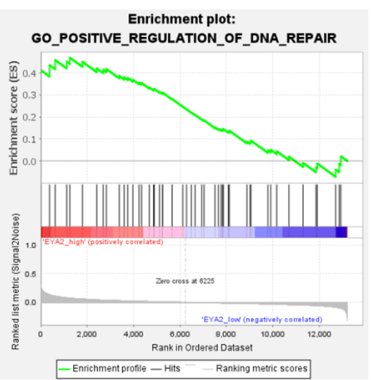

n

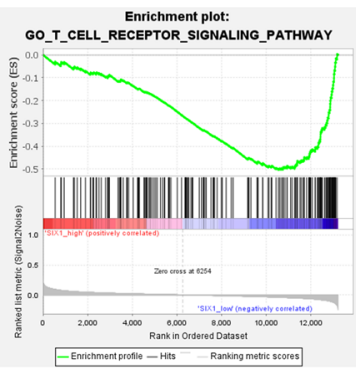

c

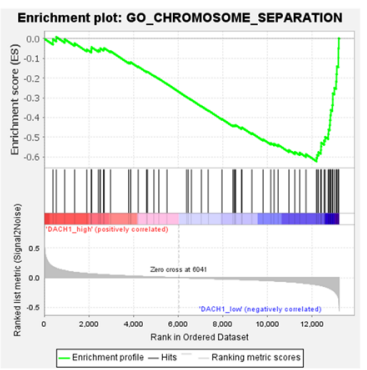

g

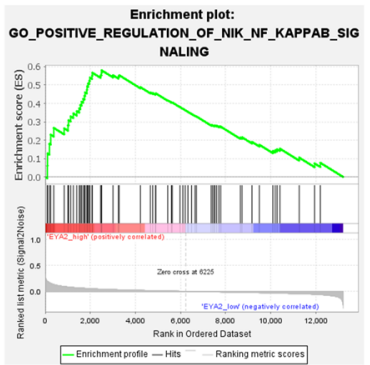

k

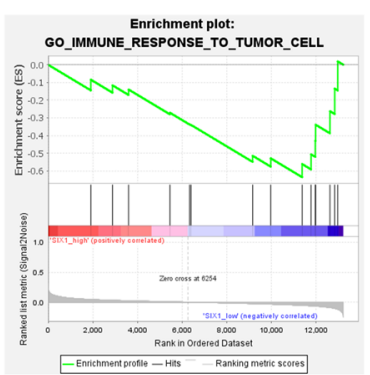

O

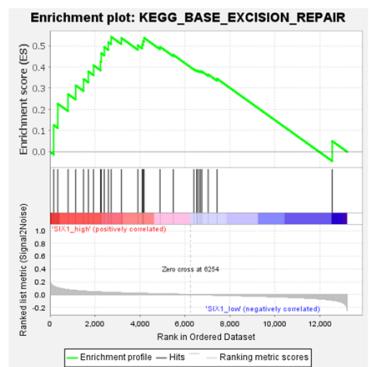

d

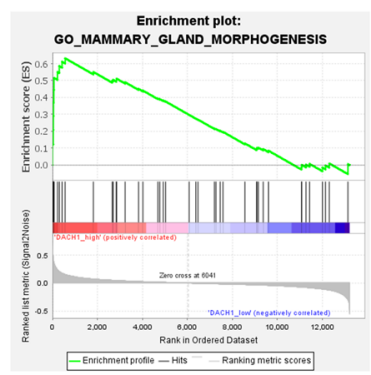

h

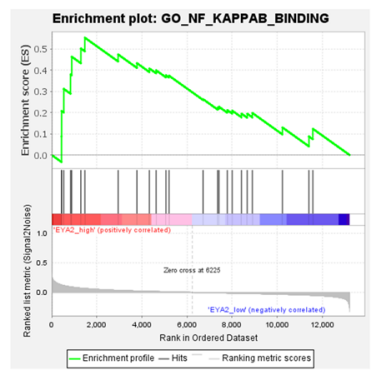

I

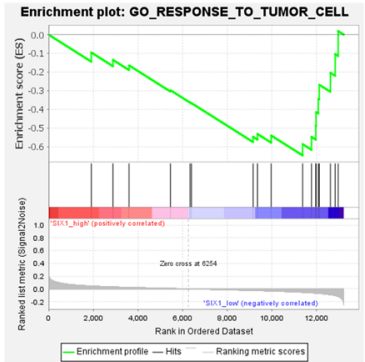

p

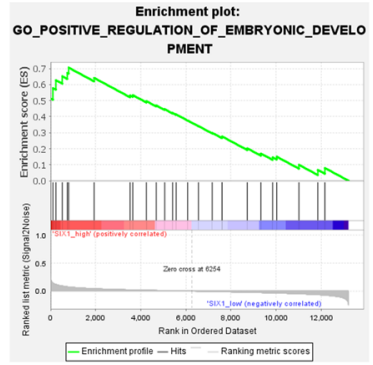

Fig. 2 The results of GSEA (GO terms). The gene profiles of samples with DACH1 low expression were significantly enriched in cell cycle G1-S transition (a), meiotic chromosome separation (b) and chromosome separation (c); The gene profiles of samples with DACH1 high expression were significantly enriched in mammary gland morphogenesis (d); The gene profiles of samples with EYA2 high expression were significantly enriched in (positive regulation) cell cycle G1-S transition (e and $\mathbf{f}$ ), (positive regulation) NF-KB signaling pathway ( $\mathbf{g}$ and $\mathbf{h}$ ); positive regulation of Wnt pathway (i) and positive regulation of DNA repair pathway (j); The gene profiles of samples with SIX1 low expression were significantly enriched in (immune) response to tumor (k and $\mathbf{I})$, positive regulation of cell killing $(\mathbf{m})$, and T cell receptor pathway $(\mathbf{n})$; The gene profiles of samples with SIX 1 high expression were significantly enriched in base excision repair (o) and embryonic development (p) 
$\mathbf{a}$

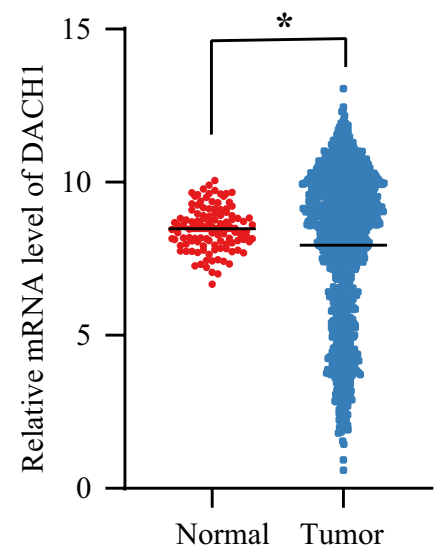

c

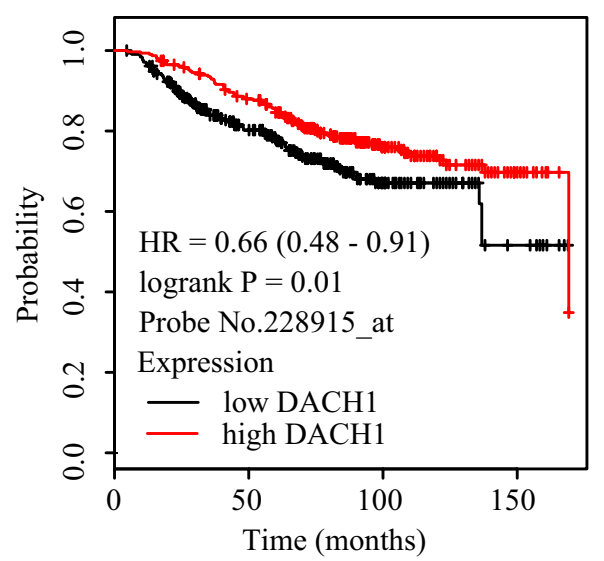

$\mathbf{e}$

Distance metastasis-free survival

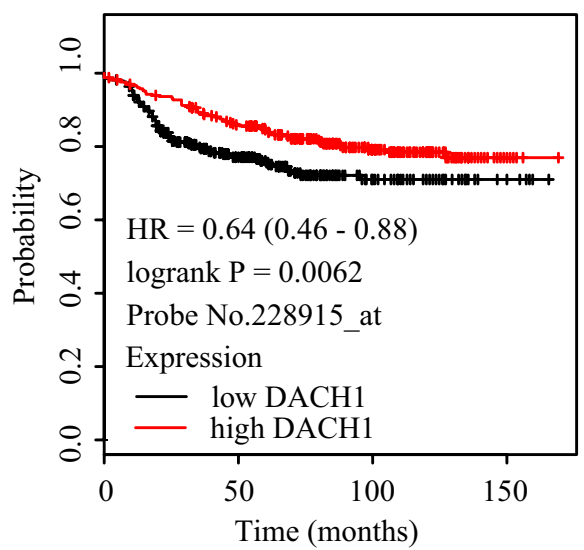

b

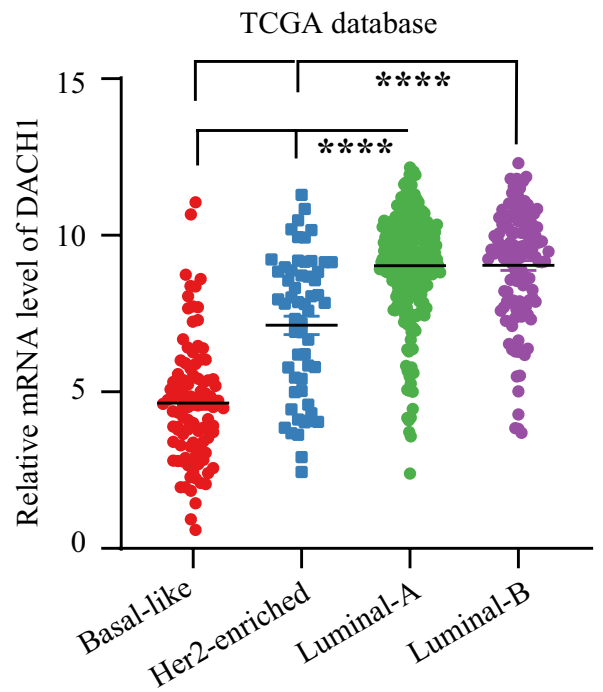

d

Relapse-free survival

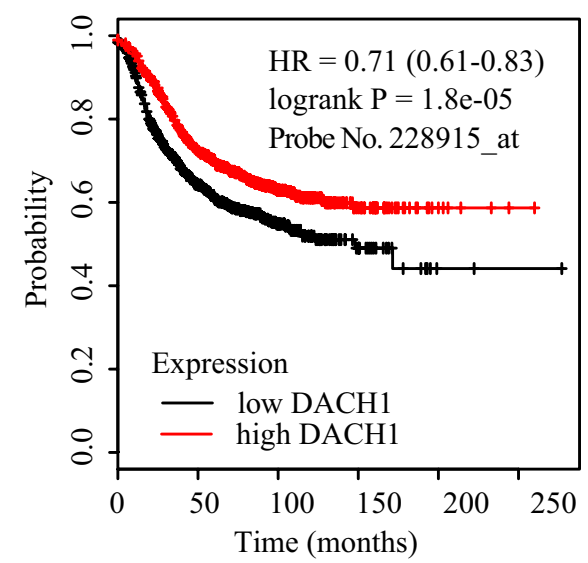

f

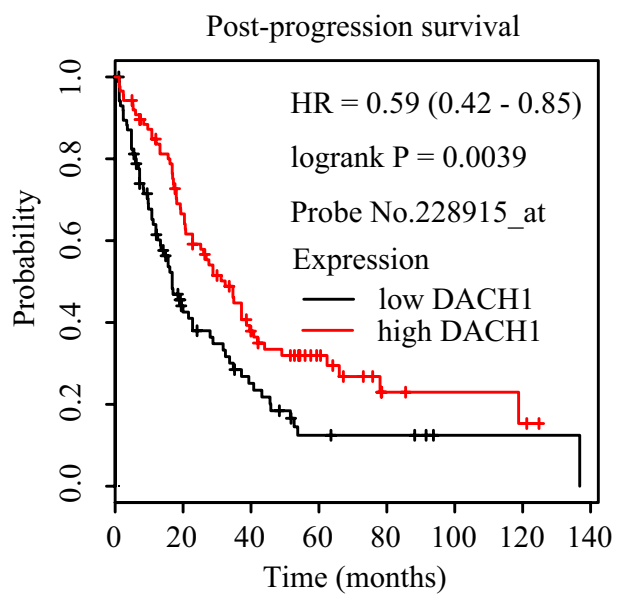

Fig. 3 The relationship between DACH1 and breast cancer. a DACH1 is downregulated in breast cancer compared with normal tissues; $\mathbf{b}$ DACH1 level is higher in Luminal A and B subtypes than Basal-like and Her-2 enriched subtypes; Survival curves of DACH1 high or low breast cancer patients: overall survival (OS) (c), relapse-free survival (RFS) (d), distant metastasis-free survival (DMFS) (e), and post-progression survival (PPS) (f) 

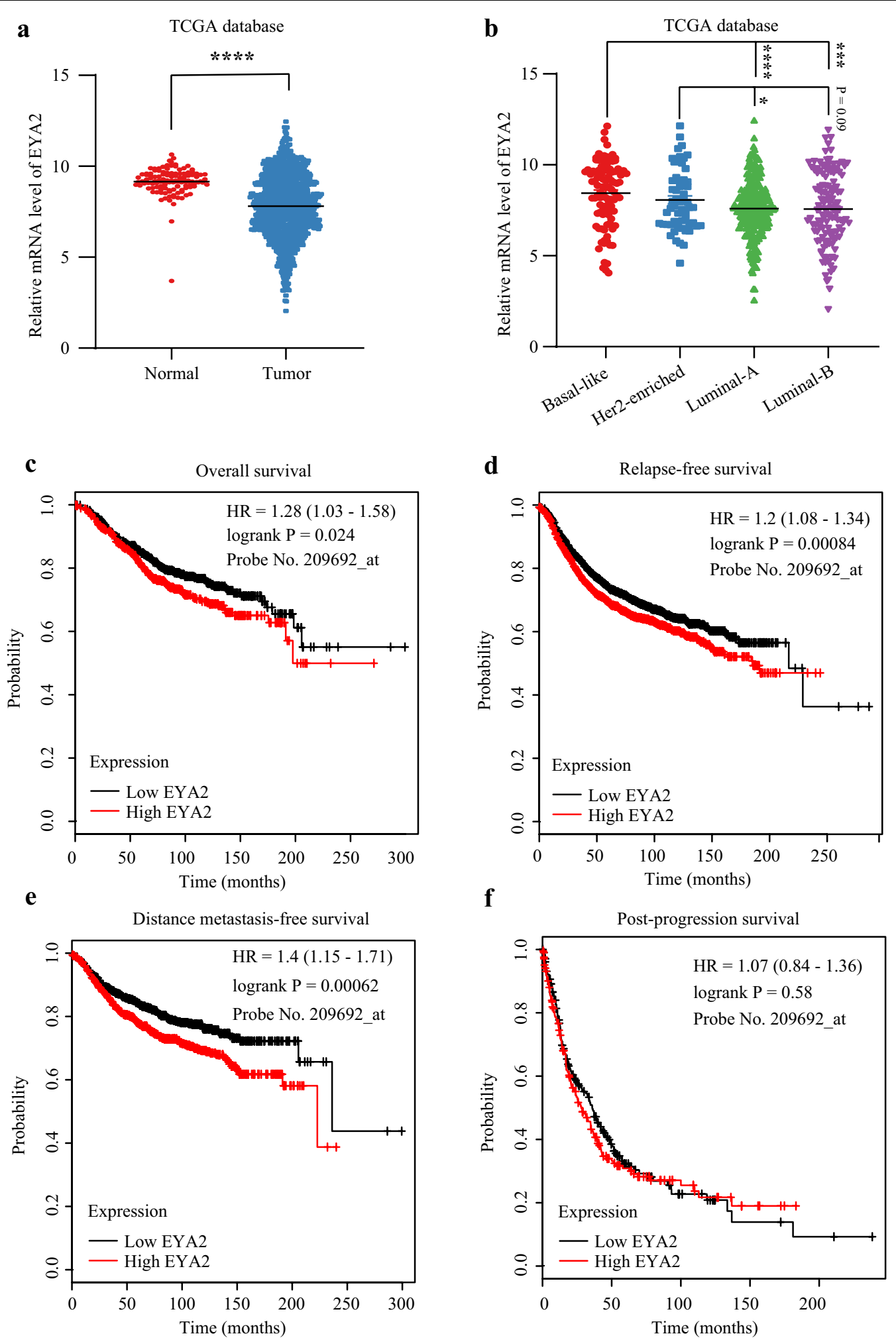

Fig. 4 The relationship between EYA2 and breast cancer. a EYA2 is lower in breast cancer compared with normal tissues; $\mathbf{b}$ EYA2 level is lower in Luminal A and B subtypes than Basal-like and Her-2 enriched subtypes; Survival curves of EYA2 high or low breast cancer patients: overall survival $(\mathrm{OS})$ (c), relapse-free survival (RFS) (d), distant metastasis-free survival (DMFS) (e), and post-progression survival (PPS) (f) 


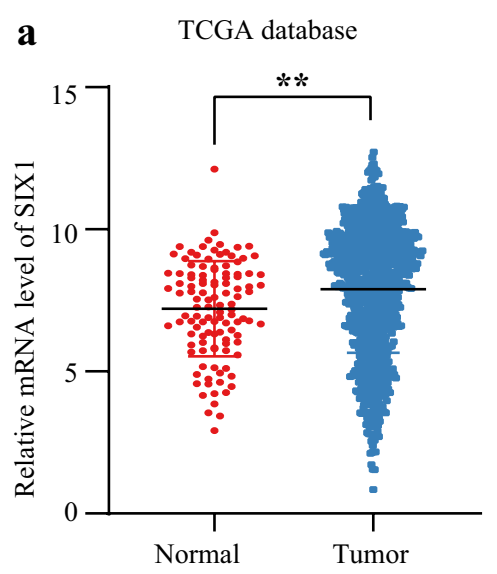

c

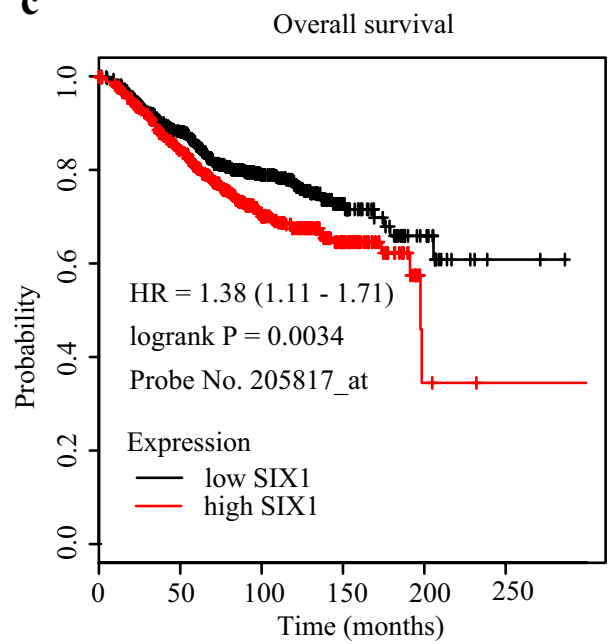

e

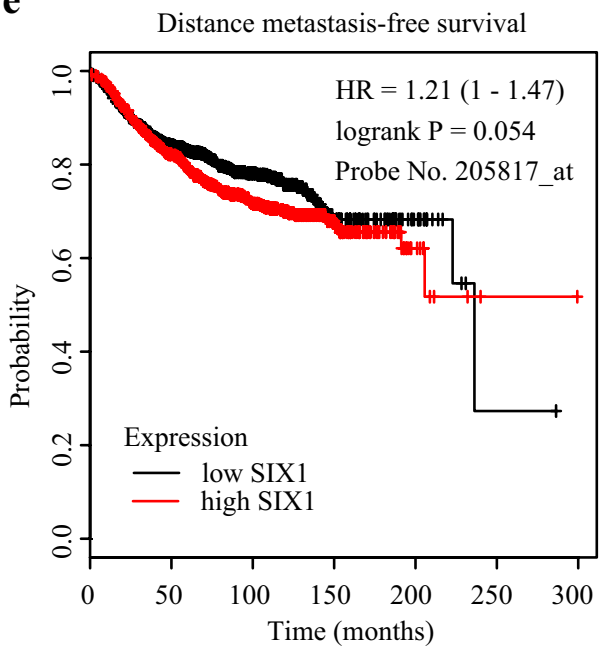

b

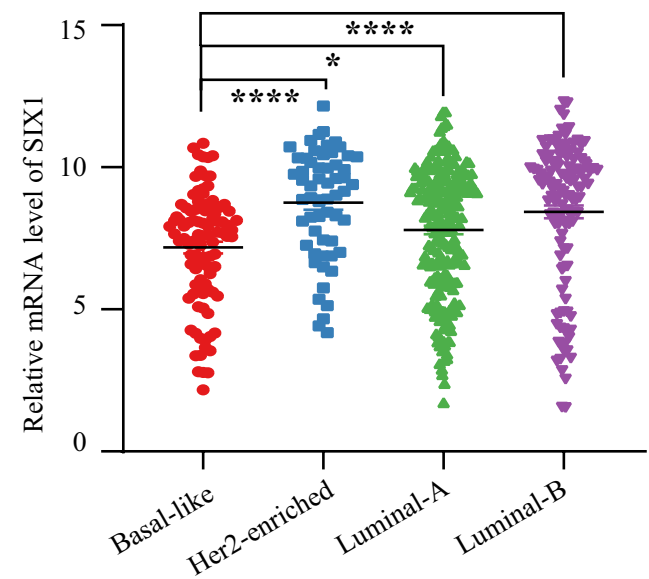

d

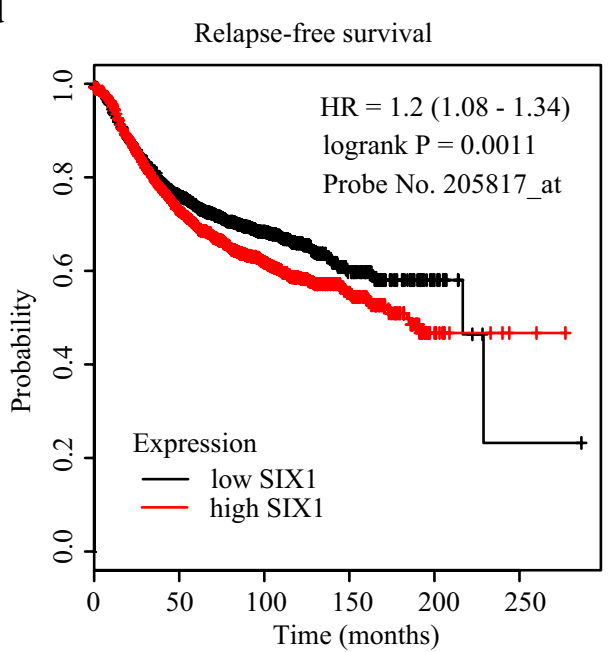

f

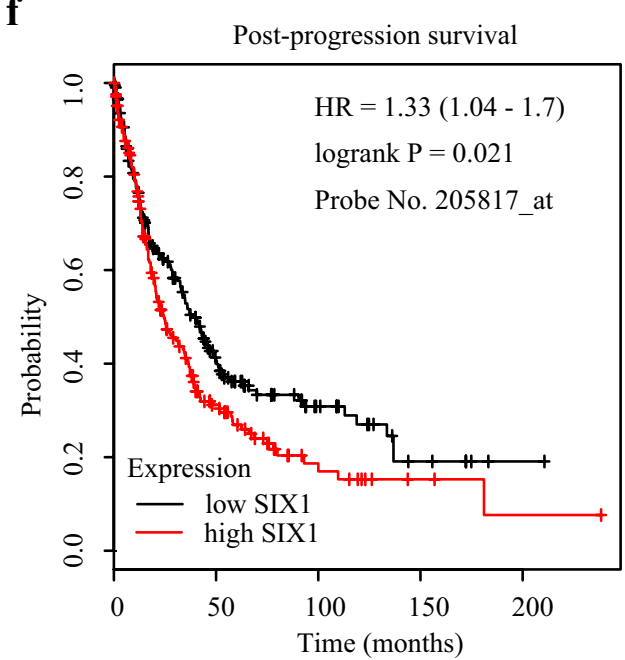

Fig. 5 The relationship between SIX 1 and breast cancer. a SIX1 is upregulated in breast cancer compared with normal tissues; $\mathbf{b}$ SIX 1 level is higher in Luminal A, B, and Her-2 enriched subtypes than Basal-like subtype; Survival curves of SIX1 high or low breast cancer patients: overall survival (OS) (c), relapse-free survival (RFS) (d), distant metastasis-free survival (DMFS) (e), and post-progression survival (PPS) (f) 
a

\begin{tabular}{|l|l|l|}
\hline Factors of univariate analysis & HR $(95 \% \mathrm{CI})$ & P value \\
\hline DACH1 & $0.758(0.554-1.037)$ & 0.083 \\
\hline EYA2 & $1.065(0.722-1.571)$ & 0.752 \\
\hline SIX1 & $1.365(1.021-1.801)$ & 0.036 \\
\hline ERBB2 & $1.168(0.864-1.579)$ & 0.313 \\
\hline ESR & $0.806(0.637-1.020)$ & 0.073 \\
\hline Cyclin E2 & $1.770(1.221-2.568)$ & 0.003 \\
\hline TOP2A & $1.990(1.335-2.924)$ & $<0.001$ \\
\hline
\end{tabular}

Index $=-0.186 * \mathrm{DACH} 1+0.287 * \mathrm{SIX} 1+0.041 *$ ERBB2 $-0.044 * \mathrm{ESR}+0.108 *$ Cyclin E2 $+0.505 * \mathrm{TOP} 2 \mathrm{~A}$

b Time dependent ROC curves

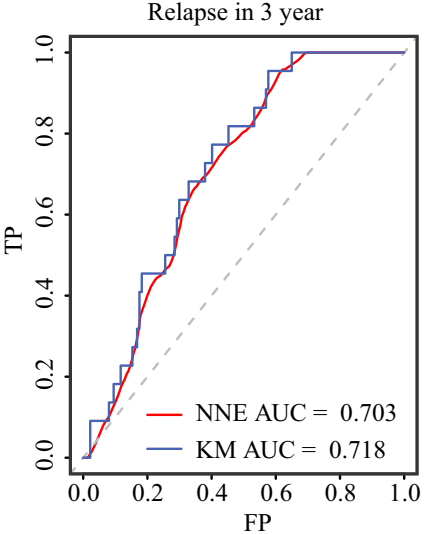

c

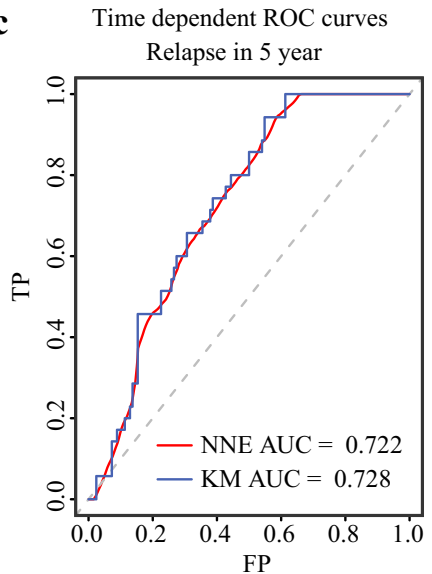

d Time dependent ROC curves Relapse in 8 year

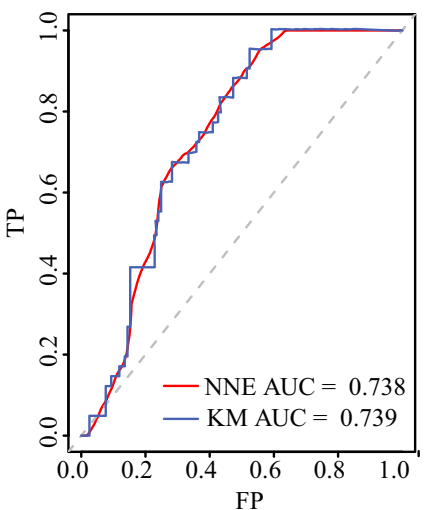

$\mathbf{e}$

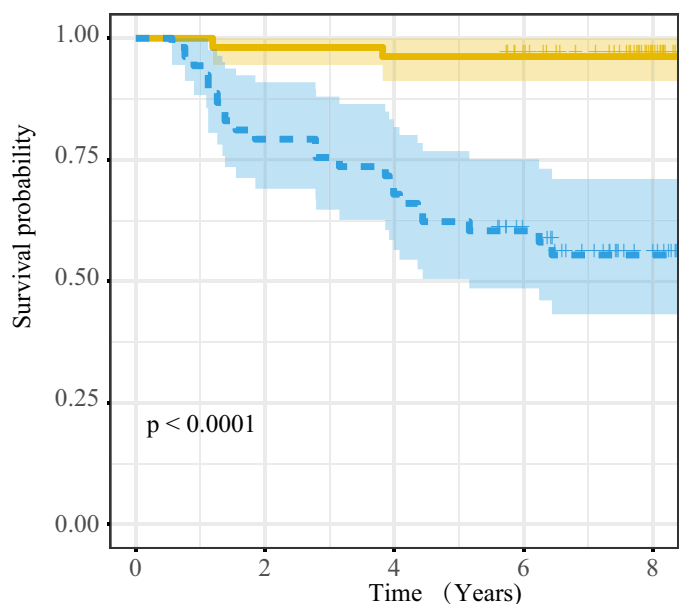

Number at risk

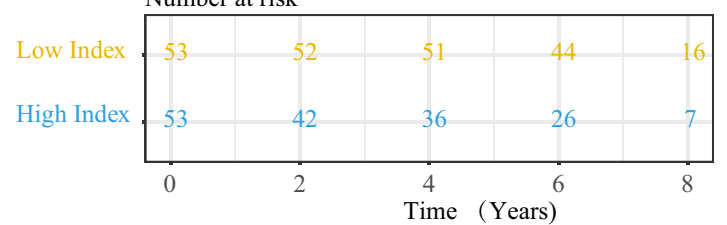

$\mathbf{f}$
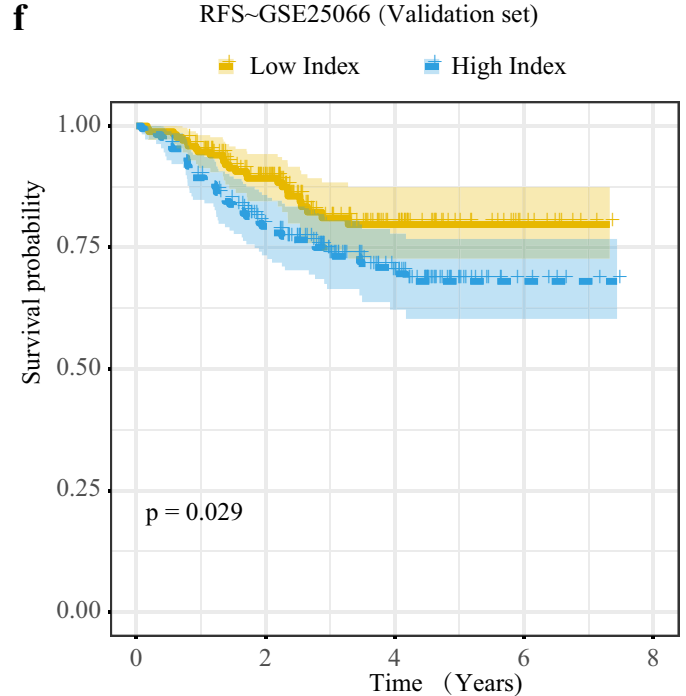

Number at risk

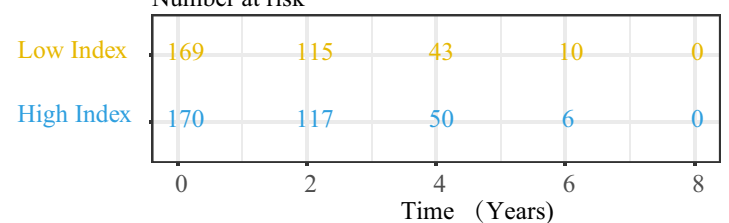

Fig. 6 The construction and verification of predictive model. a The results of Cox univariate regression analysis; The time dependent ROC curves predicting the relapse within 3 (b), 5 (c), and 8 (d) years; (e) The K-M plotter curves of patients with high or low risk index in training set GSE1456; (f) The K-M plotter curves of patients with high or low risk index in training set GSE25066 
plotter method, we found that patients with high risk index exhibited shorter RFS in GSE1456 $(\mathrm{P}<0.0001)$ (Fig. 6e). Subsequently, we utilized another dataset GSE25066 as verification. In GSE25066, patients with high risk index also had shorten RFS than individuals with low risk index $(P=0.029)$ (Fig. 6f).

\section{Discussion}

RDGN is a highly conserved signal for proper organ development across taxa [36]. However, dysregulated RGDN expression is highly related to cancer initiation and progression. For breast cancer, decreased DACH1 led to accelerated cell cycle, enhanced stemness, invasion, and metastasis. In the previous study, we found that DACH1 regulated cell cycle via inhibiting Cyclin D1 [37] and reprogramed cell stemness by downregulating stemness-associated molecules such as SOX2, Nanog, as well as KLF4 [38]. Besides, it has been verified that DACH1 could suppress epithelial-mesenchymal transition (EMT), migration, metastasis by antagonizing YB1-mediated transcriptional events, SNAI1-E-cadherin pathway, and IL-8 transcription in breast cancer cells [39-41]. Notably, it has been revealed that DACH1 was expressed in estrogen receptor breast cancer cells and acted as an endogenous inhibitor of estrogen signaling [42].

Contrary to DACH1, EYA2 facilitates proliferation, migration, invasion, and metastasis of breast cancer cells. EYA2 promoted breast cancer cellular proliferation and distant metastasis as the downstream of EGFR [28, 43]. Different from the other members of RDGN, EYA2 had a phosphatase activity. Inhibiting the phosphatase activity of EYA2 could suppress EYA2-mediated malignant biological behavior $[44,45]$. Similar to EYA2, SIX1 also play a pro-tumor role. SIX1 facilitated tumor growth by enhancing Warburg effect. Besides, SIX1 promoted EMT, metastasis, and chemotherapy resistance in breast cancer cells [46-48]. Our previous meta-analysis showed that increased SIX1 was closely related to poor prognosis of breast cancer patients [24].

In the present study, we firstly explored the effects of DACH1, EYA2, and SIX1 on downstream signaling pathways. We found that DACH1 was negatively related to cell proliferation-associated pathways while EYA2 and SIX1 were positively correlated with cell cycle or DNA replication pathways. Moreover, increased EYA2 and SIX1 were highly related with some upregulated oncogenic pathways such as Wnt and NF- $\mathrm{kB}$ pathways. It was worth mentioning that SIX1 level was positively correlated to DNA repair activity which might contribute to chemotherapy resistance in breast cancer patients with high SIX1 expression [48]. The following survival analyses showed DACH1, EYA2, and SIX1 were predictive biomarkers of prognosis of breast cancer patients. Then, we constructed a RDGN-based framework which could effectively distinguish patients with high risk of relapse.

Actually, gene expression profiling has been utilized in clinical practice for treatment decision of early stage breast cancer patients with ER positive and lymph node metastasis negative [49, 50]. Based on the results of gene expression profiling, patients could be categorized into high risk, middle risk, and low risk groups. Patients belonging to high risk group might benefit from adjuvant chemotherapy while patients belonging to low risk group could avoid unnecessary treatment [51]. Up to now, several commercial gene expression profiling tests are available including Oncotype DX (21 genes assay), Prosigna (PAM 50), MammaPrint (70 genes assay), and EndoPredict [52-54]. These test panels are usually used after breast cancer surgery with known hormone receptor and lymph node metastasis statuses. As a typical example of precious medicine, gene expression profiling is a valuable aid to guide whether or not conduct subsequent chemotherapy. Among them, Oncotype DX is the most commonly used panel [55]. In the 21 genes of Oncotype DX, 16 genes were oncogenic pathway-associated genes and 5 genes were control genes [56]. According to the results of a prospective study (NCT00310180), 93.8\% of lymph node negative, ER positive, and HER2 negative breast cancer patients with low Oncotype DX relapse scores were free of disease progression after 5 years follow-up [57]. Besides, a phase 3 trial SWOG-8814 indicated patients with high Oncotype DX relapse score could benefit from additional chemotherapy $(\mathrm{HR}=0.59$, $\mathrm{P}=0.033)$ while patients with low relapse score had no improvement in survival $(\mathrm{HR}=1.02, \mathrm{P}=0.97)$ [58]. It has been clear that patients with high risk scores benefit from additional chemotherapy. However, for predictive models such as Oncotype DX, it is still uncertain about whether patients with middle relapse scores could benefit from additional chemotherapy [59]. Our predictive model was constructed based on RDGN members which was different from commercially available predictive tests. Theoretically, this RDGN-based model would provide complementary prognostic information. This model might provide extra information for deciding personal therapeutic scheme when patients are diagnosed as middle relapse risk individuals by other commercial tests.

We hereby constructed a prognostic model to predict the outcomes of breast cancer patients. To the best of our knowledge, this is the first RDGN-based predictive model for the risk of breast cancer relapse. Despite the promising results, some questions still remain. Firstly, due to the absence of housekeeping genes, it is hard to 
set a cutoff value for other extended data sets and avoid the impact of sequencing batches. Besides, this predictive model was constructed and validated by public database and retrospective studies. This model may need to be further validated in a randomized controlled trial. Most importantly, it is unclear that this predictive model could keep its validity on other sequencing platforms. Further studies are required to resolve the problem properly.

\section{Conclusion}

Dysregulated RDGN is well known for breast cancers. Downregulated DACH1 and upregulated EYA2 and SIX1 were highly related to DNA replication and cell cycle pathways. Besides, decreased DACH1 and increased EYA2 and SIX1 heralded the poor prognosis of breast cancer patients. Combining RDGN members and other molecular biomarkers, we constructed a predictive model for the potential relapse risk. This RDGN-based model exhibited high accuracy and specificity to distinguish patients with high risk of relapse. The results of repeatability test in a verification set showed this model is stable. Generally, we believed that this model would be an important complement for commercially available predictive tests.

\section{Abbreviations \\ RDGN: Retinal determination gene network; GEO: Gene Expression Ominibus; GSEA: Gene Set Expression Analysis; TCGA: The Cancer Genome Atlas; OS: Overall survival; RFS: Relapse-free survival; DMFS: Distant metastasis-free sur- vival; PPS: Post-progression survival; EMT: Epithelial-mesenchymal transition.}

\section{Acknowledgements}

Not applicable.

\section{Authors' contributions}

$\mathrm{BD}$ and MY performed the bioinformatics analysis, drafted the manuscript and prepared the figures. AL collected the related references and participated in discussion. SL and KW designed this review and revised the manuscript. All authors contributed to this manuscript. All authors read and approved the final manuscript.

\section{Funding}

This work was supported by the National Natural Science Foundation of China (No. 81874120, 81572608), Wuhan Science and Technology Bureau (No. 2017060201010170), Natural Science Foundation of Henan (No. 162300410266, 172102410021).

\section{Availability of data and materials}

GEO datasets involved in this study could be downloaded from https://www ncbi.nlm.nih.gov/geo/. TCGA data are available at UCSC Xena (https://xena. ucsc.edu/).

\section{Ethics approval and consent to participate}

Not applicable.

\section{Consent for publication}

Not applicable.

\section{Competing interests}

The authors declare that they have no competing interests.

\section{Author details}

${ }^{1}$ Department of Molecular Pathology, The Affiliated Cancer Hospital of Zhengzhou University \& Henan Cancer Hospital, Zhengzhou 450008, China. ${ }^{2}$ Department of Oncology, Tongji Hospital of Tongji Medical College, Huazhong University of Science and Technology, Wuhan 430030, China. ${ }^{3}$ Department of Medical Oncology, The Affiliated Cancer Hospital of Zhengzhou University \& Henan Cancer Hospital, Zhengzhou 450008, China.

Received: 26 May 2020 Accepted: 4 June 2020

Published online: 15 June 2020

\section{References}

1. Siegel RL, Miller KD, Jemal A. Cancer statistics, 2019. CA Cancer J Clin. 2019;69:7-34.

2. Li N, Deng Y, Zhou L, Tian T, Yang S, Wu Y, et al. Global burden of breast cancer and attributable risk factors in 195 countries and territories, from 1990 to 2017: results from the Global Burden of Disease Study 2017. J Hematol Oncol. 2019;12:140.

3. Waks AG, Winer EP. Breast cancer treatment: a review. JAMA. 2019;321:288-300.

4. Waks AG, Winer EP. Breast Cancer Treatment. JAMA. 2019;321:316.

5. Yu S, Liu Q, Han X, Qin S, Zhao W, Li A, et al. Development and clinical application of anti-HER2 monoclonal and bispecific antibodies for cancer treatment. Exp Hematol Oncol. 2017;6:31.

6. Xu H, Yu S, Liu Q, Yuan X, Mani S, Pestell RG, et al. Recent advances of highly selective CDK4/6 inhibitors in breast cancer. J Hematol Oncol. 2017; 10:97.

7. Gusterson B, Eaves CJ. Basal-like breast cancers: from pathology to biology and back again. Stem Cell Rep. 2018;10:1676-86.

8. Li A, Yi M, Qin S, Chu Q, Luo S, Wu K. Prospects for combining immune checkpoint blockade with PARP inhibition. J Hematol Oncol. 2019;12:98.

9. Yi M, Dong B, Qin S, Chu Q, Wu K, Luo S. Advances and perspectives of PARP inhibitors. Exp Hematol Oncol. 2019;8:29.

10. Ben-Baruch NE, Bose R, Kavuri SM, Ma CX, Ellis MJ. HER2-mutated breast cancer responds to treatment with single-agent neratinib, a secondgeneration HER2/EGFR tyrosine kinase inhibitor. J Natl Compr Canc Netw. 2015:13:1061-4.

11. Bose R, Kavuri SM, Searleman AC, Shen W, Shen D, Koboldt DC, et al. Activating HER2 mutations in HER2 gene amplification negative breast cancer. Cancer Discov. 2013;3:224-37.

12. Fribbens C, O'Leary B, Kilburn L, Hrebien S, Garcia-Murillas I, Beaney M, et al. Plasma ESR1 mutations and the treatment of estrogen receptorpositive advanced breast cancer. J Clin Oncol. 2016;34:2961-8.

13. Yu S, Zhang J, Yan Y, Yao X, Fang L, Xiong H, et al. A novel asymmetrical anti-HER2/CD3 bispecific antibody exhibits potent cytotoxicity for HER2positive tumor cells. J Exp Clin Cancer Res. 2019;38:355.

14. Li X, Oghi KA, Zhang J, Krones A, Bush KT, Glass CK, et al. Eya protein phosphatase activity regulates Six1-Dach-Eya transcriptional effects in mammalian organogenesis. Nature. 2003;426:247-54.

15. Kong D, Liu Y, Liu Q, Han N, Zhang C, Pestell RG, et al. The retinal determination gene network: from developmental regulator to cancer therapeutic target. Oncotarget. 2016;7:50755-65.

16. Zheng X, Liu Q, Yi M, Qin S, Wu K. The regulation of cytokine signaling by retinal determination gene network pathway in cancer. Onco Targets Ther. 2018;11:6479-87.

17. Liu Y, Han N, Zhou S, Zhou R, Yuan X, Xu H, et al. The DACH/EYA/SIX gene network and its role in tumor initiation and progression. Int J Cancer. 2016;138:1067-75.

18. Kong D, Ma W, Zhang D, Cui Q, Wang K, Tang J, et al. EYA1 promotes cell migration and tumor metastasis in hepatocellular carcinoma. Am J Transl Res. 2019;11:2328-38.

19. Zhu H, Wu K, Yan W, Hu L, Yuan J, Dong Y, et al. Epigenetic silencing of DACH1 induces loss of transforming growth factor-beta1 antiproliferative response in human hepatocellular carcinoma. Hepatology. 2013;58:2012-22.

20. Xu H, Yu S, Yuan X, Xiong J, Kuang D, Pestell RG, et al. DACH1 suppresses breast cancer as a negative regulator of CD44. Sci Rep. 2017;7:4361. 
21. Liu Q, Li A, Tian Y, Liu Y, Li T, Zhang C, et al. The expression profile and clinic significance of the SIX family in non-small cell lung cancer. J Hematol Oncol. 2016;9:119.

22. Liang F, Lü Q, Sun S, Zhou J, Popov VM, Li S, et al. Increased expression of dachshund homolog 1 in ovarian cancer as a predictor for poor outcome. Int J Gynecol Cancer. 2012;22:386-93.

23. Zheng Y, Zeng Y, Qiu R, Liu R, Huang W, Hou Y, et al. The homeotic protein SIX3 suppresses carcinogenesis and metastasis through recruiting the LSD1/NuRD(MTA3) complex. Theranostics. 2018:8:972-89.

24. Xu HX, Wu KJ, Tian YJ, Liu Q, Han N, He XL, et al. Expression profile of SIX family members correlates with clinic-pathological features and prognosis of breast cancer: a systematic review and meta-analysis. Medicine (Baltimore). 2016;95:e4085.

25. Zhu XX, Li JH, Cai JP, Hou X, Huang CS, Huang XT, et al. EYA4 inhibits hepatocellular carcinoma by repressing MYCBP by dephosphorylating $\beta$-catenin at Ser552. Cancer Sci. 2019;110:3110-21.

26. Mo SJ, Liu X, Hao XY, Chen W, Zhang KS, Cai JP, et al. EYA4 functions as tumor suppressor gene and prognostic marker in pancreatic ductal adenocarcinoma through $\beta$-catenin/ID2 pathway. Cancer Lett. 2016;380:403-12.

27. Liu Q, Li A, Yu S, Qin S, Han N, Pestell RG, et al. DACH1 antagonizes CXCL8 to repress tumorigenesis of lung adenocarcinoma and improve prognosis. J Hematol Oncol. 2018;11:53.

28. Xu H, Jiao Y, Yi M, Zhao W, Wu K. EYA2 correlates with clinico-pathological features of breast cancer, promotes tumor proliferation, and predicts poor survival. Front Oncol. 2019;9:26.

29. Powe DG, Dhondalay GK, Lemetre C, Allen T, Habashy HO, Ellis IO, et al. $\mathrm{DACH} 1$ : its role as a classifier of long term good prognosis in luminal breast cancer. PLoS ONE. 2014;9:e84428.

30. Thomsen KG, Lyng MB, Elias D, Vever H, Knoop AS, Lykkesfeldt AE, et al. Gene expression alterations associated with outcome in aromatase inhibitor-treated ER + early-stage breast cancer patients. Breast Cancer Res Treat. 2015;154:483-94.

31. Uehiro N, Sato F, Pu F, Tanaka S, Kawashima M, Kawaguchi K, et al. Circulating cell-free DNA-based epigenetic assay can detect early breast cancer. Breast Cancer Res. 2016;18:129.

32. Hatzis C, Pusztai L, Valero V, Booser DJ, Esserman L, Lluch A, et al. A genomic predictor of response and survival following taxane-anthracycline chemotherapy for invasive breast cancer. JAMA. 2011;305:1873-81.

33. Pawitan Y, Bjöhle J, Amler L, Borg AL, Egyhazi S, Hall P, et al. Gene expression profiling spares early breast cancer patients from adjuvant therapy: derived and validated in two population-based cohorts. Breast Cancer Res. 2005;7:R953-64.

34. Subramanian A, Tamayo P, Mootha VK, Mukherjee S, Ebert BL, Gillette MA, et al. Gene set enrichment analysis: a knowledge-based approach for interpreting genome-wide expression profiles. Proc Natl Acad Sci USA. 2005;102:15545-50.

35. Nagy A, Lanczky A, Menyhart O, Gyorffy B. Validation of miRNA prognostic power in hepatocellular carcinoma using expression data of independent datasets. Sci Rep. 2018;8:9227.

36. Creed TM, Baldeosingh R, Eberly CL, Schlee CS, Kim M, Cutler JA, et al. The PAX-SIX-EYA-DACH network modulates GATA-FOG function in fly hematopoiesis and human erythropoiesis. Development. 2020;147:dev177022.

37. Wu K, Li A, Rao M, Liu M, Dailey V, Yang Y, et al. DACH1 is a cell fate determination factor that inhibits cyclin D1 and breast tumor growth. Mol Cell Biol. 2006:26:7116-29.

38. Wu K, Jiao X, Li Z, Katiyar S, Casimiro MC, Yang W, et al. Cell fate determination factor Dachshund reprograms breast cancer stem cell function. J Biol Chem. 2011;286:2132-42.

39. Wu K, Chen K, Wang C, Jiao X, Wang L, Zhou J, et al. Cell fate factor DACH1 represses YB-1-mediated oncogenic transcription and translation. Cancer Res. 2014;74:829-39.

40. Zhao F, Wang M, Li S, Bai X, Bi H, Liu Y, et al. DACH1 inhibits SNAl1-mediated epithelial-mesenchymal transition and represses breast carcinoma metastasis. Oncogenesis. 2015;4:e143.

41. Wu K, Katiyar S, Li A, Liu M, Ju X, Popov VM, et al. Dachshund inhibits oncogene-induced breast cancer cellular migration and invasion through suppression of interleukin-8. Proc Natl Acad Sci USA. 2008;105:6924-9.
42. Popov VM, Zhou J, Shirley LA, Quong J, Yeow WS, Wright JA, et al. The cell fate determination factor DACH1 is expressed in estrogen receptoralpha-positive breast cancer and represses estrogen receptor-alpha signaling. Cancer Res. 2009;69:5752-60.

43. Liang Y, Xu X, Wang T, Li Y, You W, Fu J, et al. The EGFR/miR-338-3p/EYA2 axis controls breast tumor growth and lung metastasis. Cell Death Dis. 2017;8:e2928.

44. Yuan B, Cheng L, Chiang HC, Xu X, Han Y, Su H, et al. A phosphotyrosine switch determines the antitumor activity of ERbeta. J Clin Invest. 2014;124:3378-90.

45. Krueger AB, Drasin DJ, Lea WA, Patrick AN, Patnaik S, Backos DS, et al. Allosteric inhibitors of the Eya2 phosphatase are selective and inhibit Eya2-mediated cell migration. J Biol Chem. 2014;289:16349-61.

46. Zeng J, Wei M, Shi R, Cai C, Liu X, Li T, et al. MiR-204-5p/Six1 feedback loop promotes epithelial-mesenchymal transition in breast cancer. Tumour Biol. 2016;37:2729-35.

47. Wang CA, Jedlicka P, Patrick AN, Micalizzi DS, Lemmer KC, Deitsch E, et al. SIX1 induces lymphangiogenesis and metastasis via upregulation of VEGF-C in mouse models of breast cancer. J Clin Invest. 2012;122:1895-906.

48. Li Z, Tian T, Hu X, Zhang X, Nan F, Chang Y, et al. Six 1 mediates resistance to paclitaxel in breast cancer cells. Biochem Biophys Res Commun. 2013;441:538-43.

49. McVeigh TP, Kerin MJ. Clinical use of the Oncotype DX genomic test to guide treatment decisions for patients with invasive breast cancer. Breast Cancer (Dove Med Press). 2017;9:393-400.

50. Xin L, Liu YH, Martin TA, Jiang WG. The era of multigene panels comes? The clinical utility of oncotype DX and MammaPrint. World J Oncol. 2017:8:34-40.

51. Pease AM, Riba LA, Gruner RA, Tung NM, James TA. Oncotype DX((R)) recurrence score as a predictor of response to neoadjuvant chemotherapy. Ann Surg Oncol. 2019;26:366-71.

52. Buus R, Sestak I, Kronenwett R, Denkert C, Dubsky P, Krappmann K, et al. Comparison of EndoPredict and EPclin with oncotype DX recurrence score for prediction of risk of distant recurrence after endocrine therapy. J Natl Cancer Inst. 2016;108:djw149.

53. Wallden B, Storhoff J, Nielsen T, Dowidar N, Schaper C, Ferree S, et al. Development and verification of the PAM50-based Prosigna breast cancer gene signature assay. BMC Med Genomics. 2015;8:54.

54. Groenendijk FH, Jager A, Cardoso F, van Deurzen CHM. A nationwide registry-based cohort study of the MammaPrint genomic risk classifier in invasive breast cancer. Breast. 2018;38:125-31.

55. Nicolini A, Ferrari P, Duffy MJ. Prognostic and predictive biomarkers in breast cancer: past, present and future. Semin Cancer Biol. 2018;52:56-73.

56. Curtit E, Mansi L, Maisonnette-Escot Y, Sautiere JL, Pivot X. Prognostic and predictive indicators in early-stage breast cancer and the role of genomic profiling: focus on the Oncotype DX((R)) Breast Recurrence Score Assay. Eur J Surg Oncol. 2017:43:921-30.

57. Sparano JA, Gray RJ, Makower DF, Pritchard Kl, Albain KS, Hayes DF, et al. Prospective validation of a 21-gene expression assay in breast cancer. $\mathrm{N}$ Engl J Med. 2015;373:2005-14.

58. Albain KS, Barlow WE, Shak S, Hortobagyi GN, Livingston RB, Yeh IT, et al. Prognostic and predictive value of the 21 -gene recurrence score assay in postmenopausal women with node-positive, oestrogen-receptorpositive breast cancer on chemotherapy: a retrospective analysis of a randomised trial. Lancet Oncol. 2010;11:55-65.

59. Sparano JA, Gray RJ, Makower DF, Pritchard Kl, Albain KS, Hayes DF, et al. Adjuvant Chemotherapy Guided by a 21-Gene Expression Assay in Breast Cancer. N Engl J Med. 2018;379:111-21.

\section{Publisher's Note}

Springer Nature remains neutral with regard to jurisdictional claims in published maps and institutional affiliations. 